syndrome.

Fetal valproate syndrome (FVS) and autism are reported in a 5 year-old boy exposed to valproic acid (VPA) in utero. (Williams PG, Hersh JH. Dev Med Child Neurol Sept 1997;39:632-634). The mother had taken VPA (500 mg 4 xday) for 2 to 3 years before and through the 5 th month of pregnancy. The infant's speech and language were delayed, and he communicated primarily by gestures. His head circumference was $55.5 \mathrm{~cm}$ (>95th centile), the forehead high and bossed, the nasal bridge flat, and the upper lip thin. Neurologic abnormalities included hypotonia, hyperreflexia, and ankle clonus. This is the second report of FVS manifested by autism.

\title{
LEARNING DISABILITY SUBTYPES IN NEUROFIBROMATOSIS 1
}

An analysis of neuropsychological data for 72 children, aged 6 to 18 years, with neurofibromatosis 1 (NF-1) and academic deficiencies is reported from the University of Texas MD Anderson Cancer Center, Houston, TX. Patients treated with cranial irradiation $(6 \%)$ were excluded. Those with MRI areas of hyperintensity were not excluded. The IQs of the groups analyzed ranged from 78 to 107 , and outlier groups ( $<5$ patients) with above average IQs or the mentally deficient were excluded. Three groups of academic underachievers were identified: 1) neuropsychologically normal (39\%); 2) a group with general academic deficiencies (47\%); and 3) a group with visuospatialconstructional and fine motor coordination deficiencies, and without language deficits (14\%). The low incidence of visuospatial deficiencies and absence of a group with pure language deficits were remarkable. (Brewer VR, Moore BD III, Hiscock M. Learning disability subtypes in children with neurofibromatosis. Irnl of Learning Disabilities Sept/Oct 1997;30:521-533). (Respond: Bartlett D Moore III, Division of Pediatrics (Box 87), UTMD Anderson Cancer Center, 1515 Holcombe Blvd, Houston, TX 77030).

COMMENT. By using cluster analysis and exclusion of outlier groups with above average or retarded IQ scores, children with neurofibromatosis 1 who are academic underachievers may fall into one of three groups psychologically. Approximately 40\% test normal, 50\% have general learning disabilities, and $14 \%$ have visuospatial and motor coordination problems.

Correlations of cognitive impairments with MRI abnormalities and unidentified bright objects (UBOs) are of interest. A relation between the number or location of UBOs on MRI and cognitive deficits in children with NF1 have been reported. The University of Texas study group (Moore et al, 1996) found location important, UBO hyperintensities in the thalamus correlated with a lowered IQ whereas the Johns Hopkins group (Denckla et al, 1996) found the number of locations occupied by UBOs accounted for the lowering of IQ. (See Progress in Pediatric Neurology III, PNB Publishers, 1997; p291-294, p441-442, for articles and commentary on IQ and MRI findings in NF-1).

\section{SEIZURE DISORDERS}

\section{GENETICS OF FAMILIAL INFANTILE CONVULSIONS}

Four families from northwestern France with autosomal dominant benign infantile convulsions and paroxysmal choreoathetosis were studied genetically by linkage analysis at the Wellcome Trust Centre for Human Genetics, University of Oxford, UK, and at Genetic Clinics in Amiens, France. Linkage for the disease gene was found in the pericentromeric region of 